\title{
Gleason Pattern 5 Is the Strongest Pathologic Predictor of Recurrence, Metastasis, and Prostate Cancer-Specific Death in Patients Receiving Salvage Radiation Therapy Following Radical Prostatectomy
}

\author{
William Jackson, BS'; Daniel A. Hamstra, MD, PhD'; Skyler Johnson, BS'; Jessica Zhou, MD'; Benjamin Foster, BS'; \\ Corey Foster, BS'; Darren Li, BS'; Yeohan Song, BS ${ }^{1}$; Ganesh S. Palapattu, MD²; Lakshmi P. Kunju, MD³; Rohit Mehra, MD³; \\ and Felix Y. Feng, $M D^{1}$
}

\begin{abstract}
BACKGROUND: The presence of Gleason pattern 5 (GP5) at radical prostatectomy (RP) has been associated with worse clinical outcome; however, this pathologic variable has not been assessed in patients receiving salvage radiation therapy (SRT) after a rising prostate-specific antigen level. METHODS: A total of 575 patients who underwent primary RP for localized prostate cancer and subsequently received SRT at a tertiary medical institution were reviewed retrospectively. Primary outcomes of interest were biochemical failure (BF), distant metastasis (DM), and prostate cancer-specific mortality (PCSM), which were assessed via univariate analysis and Fine and Grays competing risks multivariate models. RESULTS: On pathologic evaluation, 563 (98\%) patients had a documented Gleason score (GS). The median follow-up post-SRT was 56.7 months. A total of 60 (10.7\%) patients had primary, secondary, or tertiary GP5. On univariate analysis, the presence of GP5 was prognostic for BF (hazard ratio [HR] 3.3; $P<.0001$ ), DM (HR:11.1, $P<.0001$ ), and PCSM (HR:8.8, $P<.0001)$. Restratification of the Gleason score to include GP5 as a distinct entity resulted in improved prognostic capability. Patients with GP5 had clinically worse outcomes than patients with GS8(4+4). On multivariate analysis, the presence of GP5 was the most adverse pathologic predictor of BF (HR 2.9; $P<.0001$ ), DM (HR 14.8; $P<.0001$ ), and PCSM (HR 5.7; $P<.0001$ ). CONCLUSION: In the setting of SRT for prostate cancer, the presence of GP5 is a critical pathologic predictor of BF, DM, and PCSM. Traditional GS risk stratification fails to fully utilize the prognostic capabilities of individual Gleason patterns among men receiving SRT post-RP. Cancer 2013;119:3287-94. () 2013 American Cancer Society.
\end{abstract}

KEYWORDS: Gleason pattern, prostate cancer, salvage radiation therapy, outcomes, prognosis.

\section{INTRODUCTION}

Approximately $30 \%$ of patients undergoing radical prostatectomy (RP) for localized prostate cancer will experience biochemical recurrence. ${ }^{1,2}$ Salvage external beam radiation therapy (SRT) is known to improve various measurements of outcome, including biochemical failure (BF), distant metastasis (DM), and likely prostate cancer-specific mortality (PCSM) for those with a rising prostate-specific antigen (PSA) post-RP. ${ }^{3-6}$

The overall response rate to SRT following RP leaves room for improvement, with long-term progression-free survival estimated at less than $50 \% .{ }^{5,7}$ However, some patients obtain long-term control while others may benefit from earlier or more intensive therapy. Prognostic factors following SRT include pre-SRT PSA, use of androgen deprivation therapy (ADT), overall Gleason score (GS), extracapsular extension, surgical margins, seminal vesicle invasion, PSA doubling time (PSADT), and lymph node invasion. ${ }^{4,7}$

The presence of Gleason pattern 5 (GP5) is associated with increased recurrence and metastasis following primary treatment of localized prostate cancer with EBRT, RP, or brachytherapy. ${ }^{8-14}$ However, it is unclear what role the presence of GP5 has for patients considering SRT after biochemical recurrence post-RP. Because GP5 may be a predictor of both local and/or distant failure, analysis of its prognostic significance for those treated with SRT is a significant unmet clinical need. SRT is commonly used following biochemical recurrence after RP, and the presence or absence of GP5 could

Corresponding author: Felix Y. Feng, MD, The University of Michigan Medical Center, 1500 East Medical Center Drive, Ann Arbor, MI, 48109; Fax: (734) 936-7859; E-mail: ffeng@med.umich.edu.

${ }^{1}$ Department of Radiation Oncology, University of Michigan, Ann Arbor, Michigan; ${ }^{2}$ University of Michigan, Department of Urology, University of Michigan, Ann Arbor, Michigan; ${ }^{3}$ University of Michigan, Department of Pathology, University of Michigan, Ann Arbor, Michigan

The first two authors contributed equally to this article.

DOI: 10.1002/cncr.28215, Received: May 8, 2013; Revised: May 14, 2013; Accepted: May 21, 2013, Published online July 2, 2013 in Wiley Online Library (wileyonlinelibrary.com) 
significantly influence treatment recommendations, such as the use of pelvic $\mathrm{RT}^{15}$ and the potential use of $\mathrm{ADT}^{16}$ or other salvage therapies.

\section{MATERIALS AND METHODS}

\section{Patient Selection}

Through an institutional review board-approved retrospective analysis, 575 patients were identified who underwent primary RP for localized prostate cancer and subsequently received SRT with or without ADT between 1986 and 2010. Patients receiving SRT were identified as those receiving radiation therapy for an elevated post-RP PSA level $\geq 0.2 \mathrm{ng} / \mathrm{mL} .{ }^{17}$ Ten patients had a detectable PSA level following RP and were started on ADT prior to SRT, which resulted in an undetectable pre-SRT PSA level (represented as a pre-SRT PSA level of $0 \mathrm{ng} / \mathrm{mL}$ ). Pathologic GS was available for 563 (98\%) patients, who comprised the cohort for this analysis.

\section{Treatment}

SRT was delivered using 3-dimensional conformal or intensity-modulated radiation therapy. The range of prescribed radiation doses was 59.1-76.2 Gy, with $>95 \%$ of patients receiving 64.8-70.2 Gy. Whole-pelvic radiation therapy was used in $15.9 \%$ of patients and was usually prescribed to high-risk patients. ADT during SRT was prescribed at the treating physician's discretion based on patient risk factors.

\section{End Points}

Outcomes measured included BF, DM, and PCSM. Time to BF, DM, and PCSM were calculated from the SRT start date. BF was defined as a serum PSA level at least $0.2 \mathrm{ng} / \mathrm{mL}$ greater than the post-SRT nadir followed by a second higher serum PSA level, ${ }^{7}$ or any single level greater than $0.5 \mathrm{ng} / \mathrm{mL}$ above the post-SRT nadir. DM was defined as the presence of clinical, radiologic, or pathologic evidence of metastasis. PCSM was defined as any death in a patient with prostatic metastasis, hormone refractory disease, or death attributed to prostate cancer.

\section{Statistical Analysis}

The chi-square test and one-way analysis of variance were used to compare categorical and continuous variables, respectively. Survival and multivariate analysis were performed using the log-rank test and Kaplan-Meier methods and Fine and Grays competing risks methods, ${ }^{18}$ respectively. All statistical analyses were performed using MedCalc (version 12.3.0.0; MedCalc Software, Maria- kerke, Belgium) and Stata (version 12.1; StataCorp LP, College Station, Tex.).

\section{RESULTS}

\section{Patient Characteristics}

Patients had a median age of 65.2 years (interquartile range [IQR] 59.7-70.0), a median age-unadjusted Charlson comorbidity index of 0.0 (IQR $0-1$ ), ${ }^{19}$ and a median follow-up of 56.7 months following SRT. The median time between surgery and SRT was 30 months (IQR 1357). Patients with GP5 on average had a sorter interval between surgery and SRT than patients without GP5 (25.3 months vs 40.7 months, respectively; $P=.001$ ). The median total SRT dose was $68.4 \mathrm{~Gy}$, which was not different in patients with or without GP5 $(P=.1)$. Androgen deprivation therapy concurrent with SRT was used in $20 \%$ of all patients for a median duration of 11 months (IQR 6-24), and although patients with Gleason 8-10 disease were more likely to receive adjuvant ADT, there was no difference in either duration of ADT or in the use of ADT in patients with Gleason 8-10 with or without GP5.

Pretreatment, pathologic, and treatment-related patient characteristics as stratified by adjusted GS, accounting for the presence or absence of GP5, are shown in Table 1. Patients were stratified into 4 groups: 1 ) those with GS2-6, 2) those with GS7, 3) those with GS8 without GP5 (Gleason 4+4), and 4) those with primary, secondary, or tertiary GP5, henceforth referred to as the GP5 group. Four of the GP5 patients had primary GP5, 32 had secondary GP5, 10 had tertiary GP5, and 14 had a description of GP5 disease without notation of whether this represented the primary versus the secondary pattern. No patient had GS10 $(5+5)$. Of the patients with tertiary GP5, 1 was GS7(3+4), 6 were GS7(4+3), and 3 were GS8 $(4+4)$. There was no difference in duration of follow-up post-SRT by GS groupings $(P=.6)$.

\section{Univariate Analysis}

On univariate analysis, the traditional risk stratification of Gleason sum $(2-6,7,8-10)$ significantly predicted BF $(P=.001)$ and DM $(P=.001)$, with a trend toward predicting PCSM. Restratification of the GS with GP5 as the high-risk group significantly predicted $\mathrm{BF}, \mathrm{DM}$, and PCSM (all $P<.0001$ ). This stratification is demonstrated via Kaplan-Meier analysis in Figure 1.

There was a trend toward increased BF for those with GP5 as compared to those with GS8 $(4+4)$ (HR:1.5, $P=.07)$. However, the presence of GP5 predicted for increased DM (hazard ratio [HR]:2.2, $P=.02$ ) and 
TABLE 1. Pretreatment, Pathologic, and Treatment-Related Patient Characteristics Stratified by Gleason Score and GP5

\begin{tabular}{|c|c|c|c|c|c|c|}
\hline \multirow[b]{2}{*}{ Characteristics } & \multicolumn{4}{|c|}{ Gleason Score } & \multicolumn{2}{|c|}{$P$} \\
\hline & $2-6(n=140)$ & $7(n=295)$ & $8(4+4)(n=68)$ & GP5 (n = 60) & Overall & $\begin{array}{l}\text { GP5 vs } \\
8(4+4)\end{array}$ \\
\hline Age, y, median (IQR) & $66.1(60.9-70.2)$ & $64.2(59.4-69.8)$ & $67.6(61.8-70.8)$ & $62.8(57.8-67.3)$ & $.02^{\mathrm{a}}$ & $.6^{\mathrm{a}}$ \\
\hline CCMI, median (IQR); range & $0.0(0.0-1.0) ; 0-4.0$ & $0.0(0.0-0.0) ; 0-4.0$ & $0.0(0.0-1.0) ; 0-2.0$ & $0.0(0.0-1.0) ; 0-2.0$ & $.6^{a}$ & $.9^{\mathrm{a}}$ \\
\hline $\begin{array}{l}\text { Pre-SRT PSA, ng/mL, } \\
\text { median (IQR); range }\end{array}$ & $0.6(0.4-1.4) ; 0-27.1$ & $0.6(0.4-1.1) ; 0-11.8$ & $1.1(0.4-2.3) ; 0.1-13.6$ & 0.7 (0.3-1.5); 0.1-17.4 & $.01^{\mathrm{a}}$ & $.4^{\mathrm{a}}$ \\
\hline $\begin{array}{l}\text { Pre-SRT PSADT, mo, } \\
\text { median (IQR); range }\end{array}$ & 11.8 (7.0-21.5); 0.6-42.1 & 7.8 (4.3-15.3); 0.8-121.5 & 6.4 (3.2-13.9); 0.8-23.9 & 5.8 (4.4-13.6); 0.6-117.9 & $.2^{\mathrm{a}}$ & $.4^{\mathrm{a}}$ \\
\hline $\begin{array}{l}\text { PSA nadir post-SRT, } \\
\text { median (IQR); range }\end{array}$ & $0(0-0.1) ; 0-9.4$ & $0(0-0.3) ; 0-33.4$ & $0(0-0.2) ; 0-20.1$ & $0(0-0.2) ; 0-6.3$ & $.4^{\mathrm{a}}$ & $.6^{\mathrm{a}}$ \\
\hline SRT dose, Gy, median (IQR) & $68.4(64.8-68.4)$ & $68.4(64.8-68.4)$ & $68.4(59.1-70.8)$ & $68.4(64.8-68.4)$ & $.2^{\mathrm{a}}$ & $.1^{\mathrm{a}}$ \\
\hline Pelvic RT, \% & 14.4 & 13.3 & 26.9 & 20.0 & $.04^{\mathrm{b}}$ & $.5^{\mathrm{b}}$ \\
\hline ADT during SRT, \% & 20.7 & 15.9 & 26.5 & 33.3 & $.01^{b}$ & $.5^{\mathrm{b}}$ \\
\hline ADT duration, mo, median (range) & $8.0(5.9-14.3)$ & $12.3(6.0-23.9)$ & $10.7(5.9-23.8)$ & $9.2(5.5-23.8)$ & $.5^{\mathrm{a}}$ & $.7^{\mathrm{a}}$ \\
\hline Pathologic T stage, \% & & & & & $.03^{b}$ & $.04^{\mathrm{b}}$ \\
\hline $\mathrm{T} 1-\mathrm{T} 2 \mathrm{a}$ & 14.0 & 9.3 & 7.0 & 0 & & \\
\hline $\mathrm{T} 2 \mathrm{~b}-\mathrm{T} 2 \mathrm{c}$ & 24.7 & 25.1 & 22.8 & 13.0 & & \\
\hline T3-T4 & 61.3 & 65.6 & 70.2 & 87.0 & & \\
\hline Seminal vesicle invasion, \% & 6.6 & 13.4 & 25.0 & 45.0 & $<.0001^{\mathrm{b}}$ & $.03^{b}$ \\
\hline Positive margins, \% & 51.9 & 50.0 & 43.7 & 60.0 & $.3^{\mathrm{b}}$ & $.09^{\mathrm{b}}$ \\
\hline Extracapsular extension, \% & 42.1 & 55.6 & 58.8 & 74.6 & $.0004^{\mathrm{b}}$ & $.09^{\mathrm{b}}$ \\
\hline Lymph node involvement, \% & 4.3 & 1.2 & 7.4 & 9.1 & $.049^{\mathrm{b}}$ & $.8^{\mathrm{b}}$ \\
\hline
\end{tabular}

PCSM (HR:2.6, $P=.03$ ) when compared with patients with GS8(4+4). No difference was observed in BF, DM, or PCSM when comparing patients with primary, secondary, tertiary, and unknown GP5s on univariate analysis. The 5-year rates of BF, DM, and PCSM for patients with GP5 compared with patients with GS8(4+4) were 74\% versus $67 \%$, respectively, for BF; $44.9 \%$ versus $17.5 \%$, respectively, for $\mathrm{DM}$; and $17.7 \%$ versus $4.7 \%$, respectively, for PCSM. Although 5-year rates of BF were not statistically different for patients with GP5 compared with those with GS8 $(4+4)$, patients with GP5 were more likely to experience an early BF than GS8 $(4+4)$ patients. The median time to BF was 15.2 months (IQR 8.6-33.4) for patients with GP5 and 21.8 months (IQR 13.3-48.6) for patients with GS8 $(4+4)$. Fifty-six percent of patients with GP5 experienced BF within 18 months of SRT compared with $37 \%$ of patients with GS8 $(4+4)$.

Other variables associated with BF, DM, and PCSM on univariate analysis included pre-radiation therapy PSA level, PSADT, seminal vesicle invasion, surgical margins, and extracapsular extension (Table 2).

\section{Multivariate Analysis}

Competing risks multivariate analysis was performed using significant variables from univariate analysis. The use of concurrent ADT with SRT and whole-pelvic RT were included in the multivariate analysis to control for intensification of treatment that may have occurred for patients with GP5. The start date of SRT was likewise included to control for time-related biases in treatment and Gleason scoring. Patients receiving ADT for a persistently elevated PSA post-RP $(n=10)$ were excluded from multivariate analysis to prevent biases that may have arisen with their inclusion. PSADT was not included in the initial model, as sufficient data to calculate this variable was only available for $49 \%$ of the cohort. However, no statistically significant difference existed between those patients with and without a determinable PSADT for any of our three primary outcomes (BF, DM, PCSM, all $P>.1$ by log-rank). Thus, multivariate analysis was first performed by including all variables of interest and excluding PSADT (Table 3) and was next performed using a stepwise competing risks multivariate model, to limit overfitting of the model that included all variables (Table 4). Recognizing the importance of PSADT, a final stepwise model was created that also included pre-SRT PSADT (Table 5). Although the hazard ratios associated with the presence of GP5 were smaller in the model including PSADT, the hazard ratios for DM and PCSM were identical if the model was rerun including only the subset of 

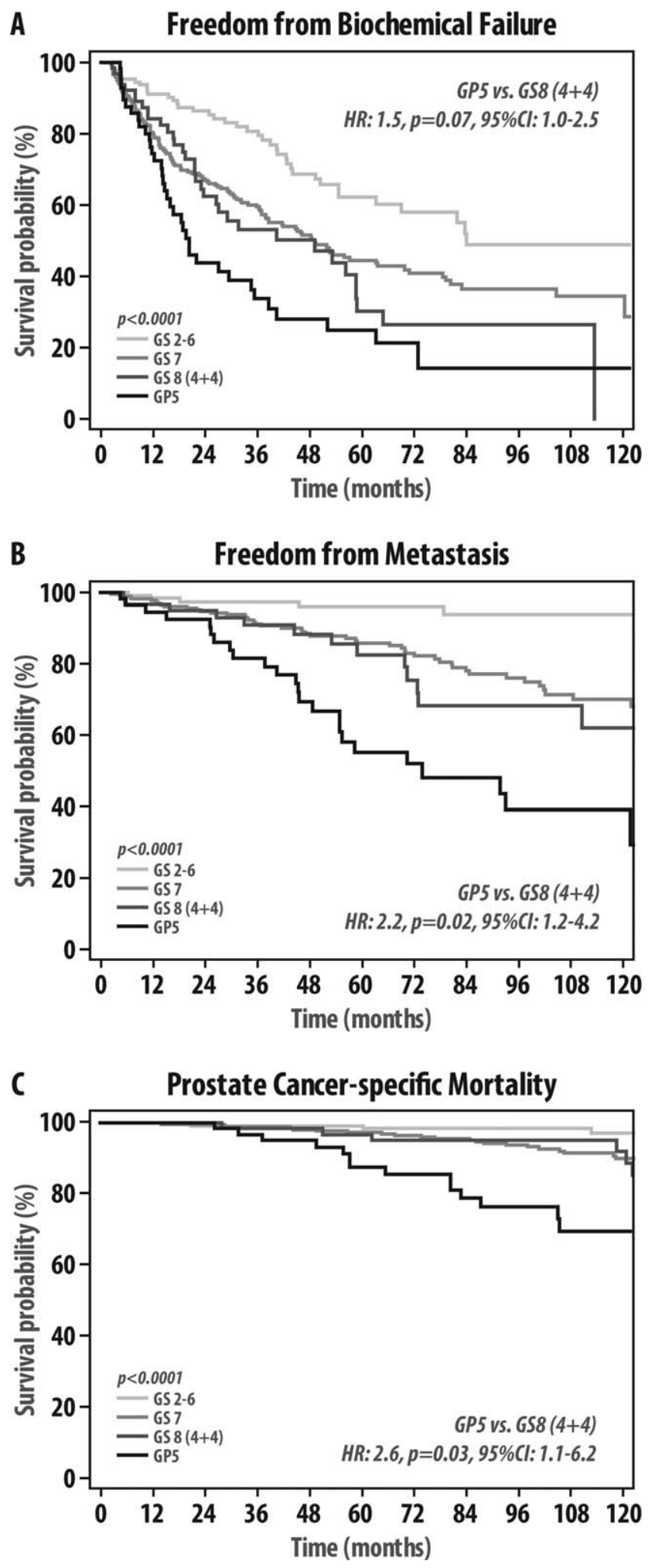

Figure 1. Freedom from biochemical failure (A), freedom from metastasis (B), and prostate cancer-specific mortality (C) for patients stratified by Gleason score and presence of Gleason pattern 5. Abbreviations: $\mathrm{Cl}$, confidence interval; GP5, Gleason pattern 5 (primary, secondary, or tertiary); GS, Gleason score; HR, hazard ratio. patients with PSADT available, but excluding PSADT as a variable in the model. This finding suggests that the smaller hazard ratios associated with GP5 in the PSADT model are a result of the smaller sample size, and not a result of the influence of PSADT.

In all models, the presence of GP5 was the most adverse pathologic predictor of BF, DM, and PCSM. Lastly, to evaluate the volume of GP5 present, an exploratory analysis was performed using a stepwise competing risks multivariate model in which the presence of GP5 was separated into 2 variables: those with primary GP5, and those with secondary or tertiary GP5. Both primary and secondary/tertiary GP5 werez significantly associated with all outcomes; however, the presence of primary GP5 was associated with a greater hazard than secondary/tertiary/unknown GP5 for BF (HR 4.0, 95\% confidence interval $[\mathrm{CI}] 1.2-12.9, P=.02$ vs HR 2.9, 95\% CI 1.7-4.8, $P<.0001$, respectively), DM (HR 22.2, 95\% CI 4.0-122.6, $P<.0001$ vs HR 14.5, 95\% CI 4.052.1, $P<.0001$, respectively), and PCSM (HR 10.6, $95 \%$ CI 2.4-47.4, $P=.002$ vs HR 5.2, 95\% CI 2.0-13.8, $P=.001$, respectively). Given that only 4 patients had primary GP5, additional analyses distinguishing between primary and secondary/tertiary GP5 were not performed.

\section{Salvage Androgen Deprivation Therapy}

Although the presence of GP5 was the most adverse pathologic predictor of poor patient outcomes on univariate and multivariate analysis, an analysis was performed to ensure that the worse outcomes were not secondary to differences in the use of salvage ADT (sADT). The time to first use of sADT following BF (Fig. 2A) and the time from the initiation of sADT to DM (Fig. 2B) were assessed for patients with and without GP5. Patients with GP5 were more likely to receive sADT earlier and more often following $\mathrm{BF}$ (HR 1.8, $P=.01$ ) than those without GP5. Likewise, patients with GP5 developed metastasis at a faster rate following the use of first sADT (HR 2.2, $P=.01$ ) than patients without GP5.

\section{DISCUSSION}

The Gleason score is one of the most critical risk-factors for prostate cancer patients treated with SRT. ${ }^{4,5,7}$ Traditional risk stratification by GS groups GS2-6, GS7, and GS8-10 has been shown to be an important prognosticator of outcomes for those receiving SRT post-RP. ${ }^{7}$ While this stratification of GS is prognostic of various outcomes for SRT, it fails to take full advantage of the individual Gleason patterns that comprise the GS. Nanda et al. demonstrated that in patients undergoing primary up-front 
TABLE 2. Univariate Survival Analysis

\begin{tabular}{|c|c|c|c|c|c|c|}
\hline & \multicolumn{2}{|c|}{ Biochemical Failure } & \multicolumn{2}{|c|}{ Distant Metastasis } & \multicolumn{2}{|c|}{$\begin{array}{c}\text { Prostate Cancer-Specific } \\
\text { Survival }\end{array}$} \\
\hline & HR (95\% Cl) & $P$ & $\mathrm{HR}(95 \% \mathrm{Cl})$ & $P$ & HR (95\% Cl) & $P$ \\
\hline Pre-SRT PSA (continuous), ng/mL & $1.1(1.06-1.13)$ & $<.0001$ & $1.1(1.1-1.2)$ & $<.0001$ & $1.1(1.0-1.2)$ & .01 \\
\hline Pre-SRT PSADT (continuous), mo & $0.96(0.94-0.98)$ & .0002 & $0.95(0.91-0.98)$ & .005 & $0.88(0.81-0.96)$ & .002 \\
\hline Any ADT during SRT & $0.8(0.6-1.1)$ & .3 & $0.9(0.5-1.4)$ & .6 & $0.7(0.5-1.7)$ & .7 \\
\hline Seminal vesicle invasion & $2.3(1.6-3.4)$ & $<.0001$ & $2.6(1.5-4.7)$ & $<.0001$ & $3.2(1.0-6.8)$ & $<.0001$ \\
\hline Positive margins & $0.75(0.6-1.0)$ & .03 & $0.8(0.5-1.2)$ & .3 & $1.15(0.7-2.0)$ & .6 \\
\hline Extracapsular extension & $1.7(1.3-2.1)$ & .0001 & $1.7(1.1-2.5)$ & .02 & $2.4(1.4-4.1)$ & .01 \\
\hline Lymph node involvement & $1.5(0.6-3.6)$ & .3 & $2.3(0.5-10.3)$ & .1 & $3.0(0.4-21.3)$ & $>.05$ \\
\hline
\end{tabular}

Abbreviations: ADT, androgen deprivation therapy; Cl, confidence interval; GP5, Gleason pattern 5; HR, hazard ratio; PSA, prostate-specific antigen; PSADT, prostate-specific antigen doubling time; SRT, salvage radiation therapy.

TABLE 3. Competing Risks Multivariate Survival Analysis Including All Variables of Interest

\begin{tabular}{|c|c|c|c|c|c|c|}
\hline \multirow[b]{2}{*}{ Variable } & \multicolumn{2}{|c|}{ Biochemical Failure } & \multicolumn{2}{|c|}{ Metastasis } & \multicolumn{2}{|c|}{$\begin{array}{c}\text { Prostate Cancer-Specific } \\
\text { Mortality }\end{array}$} \\
\hline & HR $(95 \% \mathrm{Cl})$ & $P$ & HR $(95 \% \mathrm{Cl})$ & $P$ & HR (95\% Cl) & $P$ \\
\hline \multicolumn{7}{|l|}{ Gleason Score } \\
\hline $2-6$ & Reference & & Reference & & Reference & \\
\hline 7 & $2.1(1.4-3.1)$ & $<.0001$ & $6.4(2.0-20.0)$ & .001 & $3.7(1.2-11.0)$ & .02 \\
\hline $8(4+4)$ & $2.0(1.2-3.2)$ & .004 & $6.0(1.7-20.9)$ & .01 & $3.0(0.9-10.4)$ & .08 \\
\hline GP5 & $2.9(1.7-4.7)$ & $<.0001$ & $14.4(4.3-48.8)$ & $<.0001$ & $10.8(3.1-37.9)$ & $<.0001$ \\
\hline Seminal vesicle invasion & $1.6(1.2-2.2)$ & .002 & $1.8(1.1-3.0)$ & .01 & $2.0(1.1-3.6)$ & .03 \\
\hline Extracapsular extension & $1.5(1.1-2.0)$ & .01 & $1.4(0.9-2.3)$ & .2 & $1.3(0.6-2.6)$ & .5 \\
\hline Positive margins & $0.5(0.4-0.7)$ & $<.0001$ & $0.6(0.4-0.9)$ & .04 & $0.8(0.4-1.4)$ & .4 \\
\hline Pre-SRT PSA (continuous) & $1.1(1.1-1.2)$ & $<.0001$ & $1.2(1.1-1.3)$ & $<.0001$ & $1.2(1.1-1.2)$ & $<.0001$ \\
\hline ADT with SRT duration & $0.9(0.9-0.9)$ & $<.0001$ & $0.9(0.9-0.9)$ & $<.0001$ & $0.9(0.9-1.0)$ & .2 \\
\hline Pelvic SRT & $0.7(0.5-1.1)$ & .1 & $1.1(0.6-2.1)$ & .8 & $1.5(0.7-3.2)$ & .3 \\
\hline SRT start date & $0.99(0.99-1.0)$ & .01 & $0.99(0.99-1.0)$ & .2 & $0.99(0.99-0.99)$ & $<.0001$ \\
\hline
\end{tabular}

Abbreviations: ADT, androgen deprivation therapy; Cl, confidence interval; GP5, Gleason pattern 5; HR, hazard ratio; PSA, prostate-specific antigen; SRT, salvage radiation therapy.

TABLE 4. Stepwise Competing Risks Multivariate Survival Analysis

\begin{tabular}{|c|c|c|c|c|c|c|}
\hline \multirow[b]{2}{*}{ Variable } & \multicolumn{2}{|c|}{ Biochemical Failure } & \multicolumn{2}{|c|}{ Metastasis } & \multicolumn{2}{|c|}{$\begin{array}{c}\text { Prostate Cancer-Specific } \\
\text { Mortality }\end{array}$} \\
\hline & $\mathrm{HR}(95 \% \mathrm{Cl})$ & $P$ & $\mathrm{HR}(95 \% \mathrm{Cl})$ & $P$ & $\mathrm{HR}(95 \% \mathrm{Cl})$ & $P$ \\
\hline \multicolumn{7}{|l|}{ Gleason Score } \\
\hline $2-6$ & Reference & & Reference & & Reference & \\
\hline 7 & $2.1(1.4-3.1)$ & $<.0001$ & $6.7(2.1-22.0)$ & .002 & $2.2(1.0-4.5)$ & .04 \\
\hline $8(4+4)$ & $2.0(1.2-3.2)$ & .004 & $6.3(1.7-22.7)$ & .01 & - & - \\
\hline GP5 & $2.9(1.8-4.8)$ & $<.0001$ & $14.8(4.2-52.1)$ & $<.0001$ & $5.7(2.3-14.3)$ & $<.0001$ \\
\hline Seminal vesicle invasion & $1.6(1.1-2.1)$ & .01 & $2.1(1.3-3.3)$ & .002 & $2.3(1.3-4.3)$ & .01 \\
\hline Extracapsular extension & $1.4(1.1-1.9)$ & .02 & - & - & - & - \\
\hline Positive margins & $0.5(0.4-0.7)$ & $<.0001$ & - & - & - & - \\
\hline Pre-SRT PSA (continuous) & $1.1(1.1-1.2)$ & $<.0001$ & $1.2(1.1-1.3)$ & $<.0001$ & $1.1(1.1-1.2)$ & $<.0001$ \\
\hline ADT with SRT duration & $0.9(0.9-0.9)$ & $<.0001$ & $0.9(0.9-0.9)$ & $<.0001$ & - & - \\
\hline Pelvic SRT & - & - & - & - & - & - \\
\hline SRT start date & $0.99(0.99-0.99)$ & .004 & - & - & $0.99(0.99-0.99)$ & $<.000$ \\
\hline
\end{tabular}

Abbreviations: ADT, androgen deprivation therapy; Cl, confidence interval; GP5, Gleason pattern 5; HR, hazard ratio; PSA, prostate-specific antigen; SRT, salvage radiation therapy. 
TABLE 5. Stepwise Competing Risks Multivariate Survival Analysis Including Prostate-Specific Antigen Doubling Time

\begin{tabular}{|c|c|c|c|c|c|c|c|c|c|}
\hline \multirow[b]{2}{*}{ Variable } & \multicolumn{3}{|c|}{ Biochemical Failure } & \multicolumn{3}{|c|}{ Metastasis } & \multicolumn{3}{|c|}{$\begin{array}{c}\text { Prostate Cancer-Specific } \\
\text { Mortality }\end{array}$} \\
\hline & p-value & $\mathrm{HR}$ & $95 \% \mathrm{Cl}$ & p-value & $\mathrm{HR}$ & $95 \% \mathrm{Cl}$ & p-value & $\mathrm{HR}$ & $95 \% \mathrm{Cl}$ \\
\hline \multicolumn{10}{|l|}{ Gleason Score } \\
\hline $2-6$ & Reference & & & Reference & & & Reference & & \\
\hline 7 & 0.04 & 1.5 & $1.0-2.1$ & - & - & - & - & - & - \\
\hline $8(4+4)$ & - & - & - & - & - & - & - & - & - \\
\hline GP5 & 0.002 & 2.3 & 1.4-3.8 & $<0.0001$ & 3.0 & $1.7-5.2$ & 0.02 & 3.4 & $1.4-8.4$ \\
\hline SVI & 0.02 & 1.7 & $1.1-2.9$ & 0.004 & 2.4 & $1.3-4.3$ & 0.04 & 2.4 & $1.0-5.8$ \\
\hline ECE & 0.01 & 1.6 & $1.1-2.4$ & - & - & - & - & - & - \\
\hline Positive Margins & $<0.0001$ & 0.5 & $0.4-0.7$ & - & - & - & - & - & - \\
\hline Pre-RT PSA (continuous) & $<0.0001$ & 1.3 & $1.2-1.4$ & $<0.0001$ & 1.3 & $1.2-1.5$ & $<0.0001$ & 1.2 & $1.2-1.3$ \\
\hline Pre-SRT PSADT & 0.01 & 0.9 & 0.9-0.9 & - & - & - & - & - & - \\
\hline ADT w/SRT duration & 0.001 & 0.9 & $0.9-0.9$ & 0.002 & 0.9 & $0.8-0.9$ & - & - & - \\
\hline Pelvic SRT & - & - & - & - & - & - & - & - & - \\
\hline SRT start date & - & - & - & - & - & - & 0.01 & 0.99 & $0.99-0.99$ \\
\hline
\end{tabular}

Abbreviations: ADT, androgen deprivation therapy; Cl, confidence interval; GP5, Gleason pattern 5; HR, hazard ratio; PSA, prostate-specific antigen; SRT, salvage radiation therapy.

therapy with either radiation or surgery, GS7 patients with tertiary GP5 had a similar risk of BF when compared with patients who had GS9-10, and that this risk was higher than the risk among patients with GP8 without GP5. ${ }^{13}$ Sabolch et $\mathrm{al}^{11}$ evaluated GP5 in patients treated with dose-escalated EBRT and demonstrated that the presence of GP was associated with increased DM, PCSM, and overall mortality compared to GS8 patients without GP5.

In this study, among men who underwent SRT following RP for localized prostate cancer, we demonstrated for the first time that the presence of GP5 (primary, secondary, or tertiary pattern) was the most adverse pathologic factor associated with increased BF, DM, and PCSM on univariate analysis. The increased hazard of GP5 can be most simply seen in PCSM (Fig. 1C). There was no difference in PCSM on univariate analysis for any patient with GS2 to GS8 $(4+4)(P>.05)$ with a 10-year PCSM rate of $7.7 \%$ (SEM 1.5). PCSM was substantially worse in patients with GP5 than in those with GS8 $(4+4)$ (HR 2.6, 95\% CI 1.1-6.2, $P=.03$ ) with a 10-year PCSM rate of $30.4 \%$ (SEM 7.2). Similar although less profound results were observed for metastasis (HR 2.2, 95\% CI 1.2$4.2, P=.02)$ and $\mathrm{BF}$ (HR 1.5, 95\% CI 1.0-2.5, $P=.07$ ) where in each case patients with GP5 had worse metastasis, and BF than those with GS8 $(4+4)$ while there was substantially less difference in those with GS2 through GS8(4+4).

The presence of GP5 was also correlated with other adverse pathological features, notably pathologic $\mathrm{T}$ stage and the presence of seminal vesicle invasion. However, on multivariate analysis, the presence of GP5 had the largest hazard ratio for increased risk of BF, DM, and PCSM. Additionally, in an exploratory analysis, those with primary GP5 had worse outcome than those with secondary or tertiary GP5, suggesting that increasing volumes of GP5 are associated with an increasing risk of poor clinical outcome. This finding is consistent with previous reports suggesting that an increased percentage of Gleason pattern 4 or 5 present on core biopsy or following RP may predict poorer outcomes. ${ }^{14,20}$ However, whereas patients with increasing volumes of GP5 were at greater risk of BF, $\mathrm{DM}$, and PCSM in our analysis, patients with secondary or tertiary GP5 were still at a significantly greater risk for poor outcomes following SRT than patients with GS8 $(4+4)$ or with lower GS.

It is unclear whether the increased risk of poor clinical outcome associated with GP5 is due to local, regional, or metastatic failure in patients with GP5. However, patients with GP5 received SRT sooner than patients without GP5, were more likely to have BF after SRT, and had a shorter interval from SRT to BF, where the interval to $\mathrm{BF}$ has been demonstrated to be prognostic for metastasis after RP, EBRT, brachytherapy, and SRT. ${ }^{21-24}$ As further evidence of the increased risk associated with GP5 on multivariate analysis, those with GP5 were much more likely to develop metastasis and experience prostate cancer-specific death than patients with GS8 $(4+4)$, despite the fact that both groups had similar 5-year rates of BF (74\% vs $67 \%$, respectively). Even controlling for the use of ADT either with SRT or following SRT, where those with GP5 were more likely to receive either adjuvant 


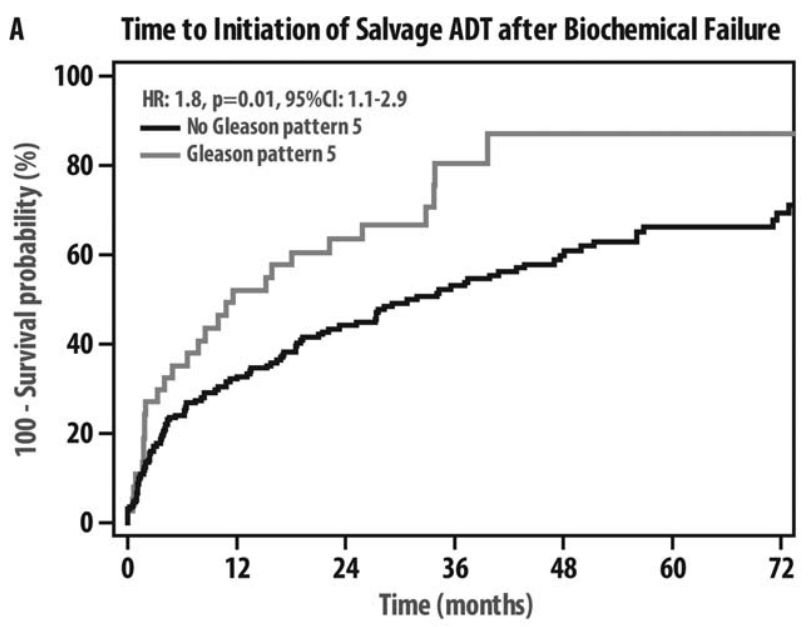

B Time from Initiation of Salvage ADT to Development of Metastasis

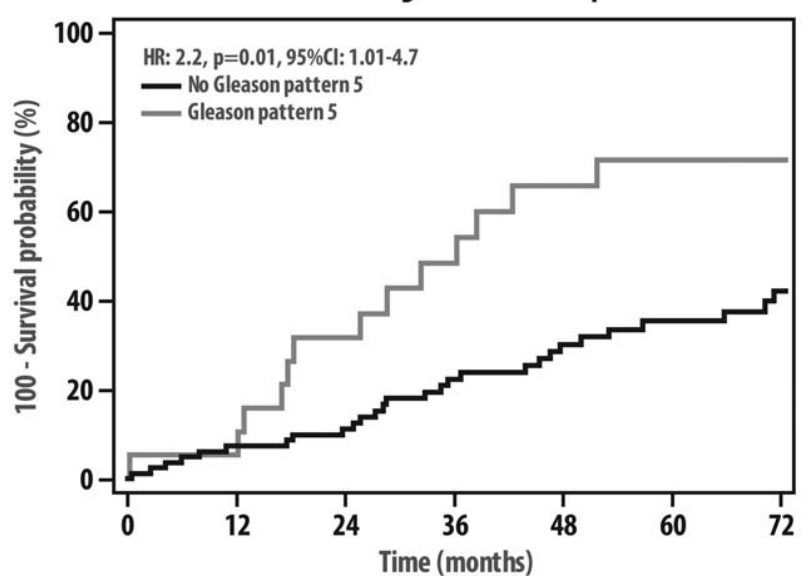

Figure 2. (A) Time to initiation of salvage androgen deprivation therapy after biochemical failure. (B) Time from initiation of salvage androgen deprivation therapy to the development of metastasis. Abbreviations: ADT, androgen deprivation therapy; $\mathrm{Cl}$, confidence interval; HR, hazard ratio.

or salvage ADT, those with GP5 still progressed to DM earlier, which would be consistent with GP5 resulting in higher metastatic potential and also potentially with GP5 being associated with early conversion to androgen independence. ${ }^{25}$

Pathologically, GP5 is defined as prostate adenocarcinoma with essentially no glandular differentiation, composed of solid sheets, cords, or single cells; comedocarcinoma with central necrosis surrounded by papillary cribiform or solid masses, ${ }^{26}$ thus signifying an undifferentiated state. This undifferentiated state may represent one that has lost biological regulation of the androgen pathway, which could explain our findings regarding sADT, as well as potentially the aggressive phenotype. With the advent of increased understanding of the biological changes that drive prostate cancer, future research should aim to define the differences in molecular pathways driving GP5 disease versus non-GP5 disease. The ability to highlight the underlying genetic changes that drive the formation of GP5 disease through advances in genomics and transcriptome analysis may allow for creation of targeted therapies that improve outcomes for patients with GP5 prostate cancer.

Given the poorer prognosis associated with the presence GP5 in this study, treatment intensification may be a reasonable option for patients undergoing RP who are found to harbor GP5. The fact that the presence of GP5 was independently predictive of DM and PCSM suggests a need for earlier treatment to prevent seeding of metastasis, or if earlier treatment is not provided, the addition of systemic therapy to SRT to account for the increased risk that micrometastasis may already exist. ${ }^{16}$ However, it must be noted that the presence of GP5 does not serve as an absolute contraindication to SRT. Whereas patients with GP5 have a higher likelihood of not responding to SRT than those without GP5, those with localized GP5 recurrences constitute the group of patients for whom SRT may have the potential to provide the greatest benefit. This notion that aggressive prostate cancer recurrences benefit most from SRT is supported by analyses performed by Cotter et $\mathrm{al}^{3}$ and Trock et al, ${ }^{6}$ where in both cases SRT was associated with improved survival in patients with BF after RP compared with observation and the benefit of SRT was greatest in those with the highest-risk disease. In fact, in our present analysis, approximately $40 \%$ of patients with GP5 had not developed metastasis 10 years following SRT, and greater than $65 \%$ of patients were still alive.

The major limitations of the present analysis include the retrospective nature, which necessitates external validation of these findings, and the long period over which the cohort received treatment. The Gleason scoring system has undergone various changes since its inception, most recently with the changes recommended by the 2005 International Society of Urological Pathology consensus conference. ${ }^{26}$ However, the majority of changes over the past decade have revolved around GP4, whereas the definition of GP5 has remained relatively stable. We attempted to control for potential changes in the Gleason scoring system as well as changes in treatment approaches that may have occurred over the study period by including the start date of SRT in the multivariate analysis. The start date of SRT was significantly associated with PCSM, although the HR (0.99) of approximately 1 resulted in a minor protective association with more recent SRT start dates, which had minimal impact upon the overall analysis. 
In conclusion, we for the first time demonstrate that the presence of GP5 is critically associated with clinically significant outcomes in the setting of SRT following RP. GP5 was the most adverse pathologic risk factor on univariate and multivariate analysis, despite the earlier and more prevalent use of sADT in these patients. Additionally, restratification of the Gleason scoring system with the presence of GP5 defining the highest-risk group was a better predictor of patient outcomes than the traditional Gleason scoring system. Clearly, patients with GP5 do not have the same clinical prognosis as patients with GS8 $(4+4)$, and thus the two should not be considered as one entity when stratifying patient risk by Gleason score. ${ }^{7,17}$ Future risk stratification schemes and predictive nomograms for SRT candidates following RP for prostate cancer should account for the presence of GP5, as the results here suggest that this one factor could have a greater influence on prognostic tools than any other variable. Intensification of treatment regimens, with initiation of adjuvant therapy following RP or the concurrent use of systemic therapies at the time of SRT may be reasonable options for patients with GP5 following radical prostatectomy.

\section{FUNDING SOURCES}

No specific funding was disclosed.

\section{CONFLICT OF INTEREST DISCLOSURES}

The authors made no disclosures.

\section{REFERENCES}

1. Roehl KA, Han M, Ramos CG, Antenor JAV, Catalona WJ. Cancer progression and survival rates following anatomical radical retropubic prostatectomy in 3,478 consecutive patients: long-term results. J Urol. 2004;172:910-914.

2. Han M, Partin AW, Pound CR, Epstein JI, Walsh PC. Long-term biochemical disease-free and cancer-specific survival following anatomic radical retropubic prostatectomy. The 15-year Johns Hopkins experience. Urol Clin North Am. 2001;28:555-565.

3. Cotter SE, Chen MH, Moul JW, et al. Salvage radiation in men after prostate-specific antigen failure and the risk of death. Cancer. 2011;117:3925-3932.

4. Katz MS, Zelefsky MJ, Venkatraman ES, Fuks Z, Hummer A, Leibel SA. Predictors of biochemical outcome with salvage conformal radiotherapy after radical prostatectomy for prostate cancer. J Clin Oncol. 2003;21:483-489.

5. Goenka A, Magsanoc JM, Pei X, et al. Long-term outcomes after high-dose postprostatectomy salvage radiation treatment. Int J Radiat Oncol Biol Phys. 2012;84:112-118.

6. Trock BJ, Han M, Freedland SJ, et al. Prostate cancer-specific survival following salvage radiotherapy vs observation in men with biochemical recurrence after radical prostatectomy. JAMA. 2008;299: 2760-2769.

7. Stephenson AJ, Scardino PT, Kattan MW. Predicting the outcome of salvage radiation therapy for recurrent prostate cancer after radical prostatectomy. J Clin Oncol. 2007;25:4153-4153.
8. Hashine K, Yuasa A, Shinomori K, Shirato A, Ninomiya I, Teramoto N. Tertiary Gleason pattern 5 and oncological outcomes after radical prostatectomy. Jpn J Clin Oncol. 2011;41:571-576.

9. Trock BJ, Guo CC, Gonzalgo ML, Magheli A, Loeb S, Epstein JI. Tertiary Gleason patterns and biochemical recurrence after prostatectomy: proposal for a modified Gleason scoring system. J Urol. 2009; 182:1364-1370.

10. Stock RG, Cesaretti JA, Hall SJ, Stone NN. Outcomes for patients with high-grade prostate cancer treated with a combination of brachytherapy, external beam radiotherapy and hormonal therapy. BJU Int. 2009;104:1631-1636.

11. Sabolch A, Feng FY, Daignault-Newton S, et al. Gleason pattern 5 is the greatest risk factor for clinical failure and death from prostate cancer after dose-escalated radiation therapy and hormonal ablation. Int J Radiat Oncol Biol Phys. 2011;81:e351-e360.

12. Vis AN, Roemeling S, Kranse R, Schroder FH, van der Kwast TH. Should we replace the Gleason score with the amount of high-grade prostate cancer? Eur Urol. 2007;51:931-939.

13. Nanda A, Chen MH, Renshaw AA, D'Amico AV. Gleason Pattern 5 prostate cancer: further stratification of patients with high-risk disease and implications for future randomized trials. Int J Radiat Oncol Biol Phys. 2009;74:1419-1423.

14. Cheng L, Koch MO, Juliar BE, et al. The combined percentage of Gleason patterns 4 and 5 is the best predictor of cancer progression after radical prostatectomy. J Clin Oncol. 2005;23:2911-2917.

15. Spiotto MT, Hancock SL, King CR. Radiotherapy after prostatectomy: improved biochemical relapse-free survival with whole pelvic compared with prostate bed only for high-risk patients. Int J Radiat Oncol Biol Phys. 2007;69:54-61.

16. Shipley WU, Hunt D, Lukka HR, et al. Initial report of RTOG 9601, a phase III trial in prostate cancer: Effect of anti-androgen therapy (AAT) with bicalutamide during and after radiation therapy (RT) on freedom from progression and incidence of metastatic disease in patients following radical prostatectomy (RP) with pT2-3,N0 disease and elevated PSA levels. J Clin Oncol. 2011;29:1.

17. Cooperberg MR, Hilton JF, Carroll PR. The CAPRA-S score: a straightforward tool for improved prediction of outcomes after radical prostatectomy. Cancer. 2011;117:5039-5046.

18. Fine JP, Gray RJ. A proportional hazards model for the subdistribution of a competing risk. J Am Stat Assoc. 1999;94:496-509.

19. Charlson ME, Pompei P, Ales KL, MacKenzie CR. A new method of classifying prognostic comorbidity in longitudinal studies: development and validation. J Chronic Dis. 1987;40:373-383.

20. D'Ambrosio DJ, Hanlon AL, Al-Saleem T, et al. The proportion of prostate biopsy tissue with Gleason pattern 4 or 5 predicts for biochemical and clinical outcome after radiotherapy for prostate cancer. Int J Radiat Oncol Biol Phys. 2007;67:1082-1087.

21. Shilkrut M, McLaughlin W, Merrick GS, et al. Interval to biochemical failure predicts clinical outcomes in patients with high-risk prostate cancer treated by combined-modality radiotherapy [published online ahead of print May 9, 2013]. Int J Radiat Oncol Biol Phys. doi: 10.1016/j.ijrobp.2013.03.028.

22. Buyyounouski MK, Hanlon AL, Horwitz EM, Pollack A. Interval to biochemical failure highly prognostic for distant metastasis and prostate cancer-specific mortality after radiotherapy. Int J Radiat Oncol Biol Phys. 2008;70:59-66.

23. Freedland SJ, Humphreys EB, Mangold LA, et al. Risk of prostate cancer-specific mortality following biochemical recurrence after radical prostatectomy. JAMA. 2005;294:433-439.

24. Johnson S, Jackson W, Li D, et al. The interval to biochemical failure is prognostic for metastasis, prostate cancer-specific mortality, and overall mortality after salvage radiation therapy for prostate cancer. Int J Radiat Oncol Biol Phys. 2013;86:554-561.

25. Benaim EA, Pace CM, Roehrborn CG. Gleason score predicts androgen independent progression after androgen deprivation therapy. Eur Urol. 2002;42:12-17.

26. Epstein JI, Allsbrook WC Jr, Amin MB, Egevad LL. The 2005 International Society of Urological Pathology (ISUP) Consensus Conference on Gleason Grading of Prostatic Carcinoma. Am J Surg Pathol. 2005;29:1228-1242. 